\title{
STAT3 influences the characteristics of stem cells in cervical carcinoma
}

\author{
HUA WANG, JIE DENG, HONG-YING REN, PING JIA, WEI ZHANG, \\ MING-QUN LI, SHU-WEI LI and QING-HONG ZHOU \\ Department of Obstetrics and Gynecology, Xiangyang No. 1 People's Hospital, \\ Hubei University of Medicine, Xiangyang, Hubei 441000, P.R. China
}

Received February 27, 2017; Accepted May 29, 2017

DOI: $10.3892 / 01.2017 .6454$

\begin{abstract}
The purpose of the study was to investigate the role of the signal transducer and activator of transcription 3 (STAT3), signal transduction protein in regulating the biological characteristics of stem cells in cervical carcinoma. Overexpressed plasmid of STAT3 was constructed and used to transfect SiHa into cervical carcinoma cells. STAT3-targeted specific siRNA was designed and produced. The effects of STAT3 upregulation (or inhibition) on the expression of NANOG, OCT4 and SOX2 markers of stem cells were measured, using western blot analysis and RT-qPCR. In addition, the tumor sphere experiment was also conducted to detect the formation of tumor spheres after the intervention of expression of STAT3 and the expression of STAT3, NANOG, OCT4 and SOX2 was detected in 35 cases of cervical carcinoma tissues and 31 cases of normal cervical tissues using immunohistochemistry. We determined whether the STAT3 overexpression plasmid was successfully constructed using enzyme digestion, PCR for bacterium solution, western blot analysis and RT-qPCR and found that the plasmid met the requirements of subsequent procedures. Compared with the empty plasmid group and STAT3 low expression group, the mRNA and protein expression of markers of stem cells, OCT4, SOX2 and NANOG were significantly elevated in the STAT3 overexpression group with statistically significant differences $(\mathrm{P}<0.05)$, the formation ratio of tumor spheres in the STAT3 overexpression group was also significantly higher than those in the other two groups $(\mathrm{P}<0.05)$. The positive expression of STAT3, OCT4, NANOG and SOX2 in the cervical squamous carcinoma group was also markedly higher than that in the chronic cervicitis group $(\mathrm{P}<0.05)$. This study led us to a conclusion that STAT3 can regulate the characteristics of stem cells in cervical carcinoma, and STAT3, NANOG, OCT4 and SOX2
\end{abstract}

Correspondence to: Dr Jie Deng, Department of Obstetrics and Gynecology, Xiangyang No. 1 People's Hospital, Hubei University of Medicine, 15 Jiefang Road, Xiangyang, Hubei 441000, P.R. China E-mail: dp712i@163.com

Key words: cervical carcinoma, signal transducer and activator of transcription 3, stem cell are highly expressed in cervical squamous carcinoma, thus able to promote the progression of cervical carcinoma.

\section{Introduction}

With the more detailed studies on the stem cells in cervical carcinoma, we have successfully isolated and identified the stem cells in cervical carcinoma from multiple types of cervical carcinoma tissues and cell strains, and some scholars have proposed that tumor stem cells in cervical carcinoma are involved in the formation, infiltration, metastasis, chemotherapy tolerance and recurrence of cervical carcinoma $(1,2)$. Tumor stem cells are characterized with the features of self-renewal, infinite proliferation and the potential of multiorientated differentiation, which may be realized by the regulation of many development-related signal transduction factors and transcriptional activators (3-5).

The signal transducer and activator of transcription 3 (STAT3), as a kind of functional protein coupled with the tyrosine phosphorylation signaling pathway (6-9), exerts an important effect on regulating the expression of proteins relating to various functions, such as cell proliferation, differentiation, apoptosis and immune escape (10). Active STAT3 is a key to the malignant changes of cells and the abnormal high expression or activity enhancement of STAT3 has been identified in a variety of tumor cell strains and tumor tissues such as the cervical carcinoma tissues and HeLa cell strains (7,11-13). Suppression on the expression of STAT3 gene can inhibit cell proliferation and induce cell apoptosis in HeLa strains of cervical carcinoma.

$N A N O G$, a kind of gene specifically expressed in the embryonic stem cells $(14,15)$. It is a key gene capable of maintaining the self-renewal, proliferative and sub-totipotent capability of stem cells, and together with other key pluripotent factors, such as OCT4 and SOX2 it can control the expression of a series of target genes (16-18) that play key roles in sustaining the pluripotency of embryonic stem cells. These factors can form a regulatory network to support or limit the expression levels mutually $(19,20)$.

Although it has been found that STAT3 signal transduction factor is indispensable for the proliferation of tumor stem cells, it can upregulate the expression of NANOG, transcription factor of stem cells, by binding OCT3/4, to further maintain 
the self-renewal capability of stem cells $(21,22)$. However, it remains unknown currently whether STAT3 can regulate the characteristics of stem cells of cervical carcinoma through the regulations of markers of stem cells, such as NANOG, OCT4 and SOX2. Thus, after intervention of the STAT3 expression, we assayed the changes in the expression of markers of stem cells, i.e., NANOG, OCT4 and SOX2, using western blotting and RT-qPCR and observed the formation of tumor sphere through the tumor sphere experiment and detected the expression of STAT3, NANOG, OCT4 and SOX2 in clinical samples using immunohistochemistry.

\section{Materials and methods}

Samples. SiHa cell strains of human cervical carcinoma were preserved in the Scientific Research Center of Zhongnan Hospital of Wuhan University; rabbit anti-human STAT3, OCT4, SOX2 and NANOG polyclonal antibodies were purchased from Abcam (Cambridge, UK), DMEM high-sugar medium was purchased from Hyclone (Logan, UT, USA), $10 \%$ fetal bovine serum was from Gibco (Grand Island, NY, USA), Lipofectamine 2000 was from Invitrogen Life Technologies (Carlsbad, CA, USA). The cervical carcinoma tissue samples were collected from 76 patients who were admitted to the Department of Obstetrics and Gynecology, Xiangyang No. 1 People's Hospital, Hubei University of Medicine for surgery between January 2013 and February 2014. Of the samples 35 were cervical carcinoma and 31 were chronic cervicitis. All patients gave their informed consent, and after surgery, the samples were immediately preserved at $-70^{\circ} \mathrm{C}$ after being processed using liquid nitrogen. This study was approved by the Ethics Committee of Xiangyang No. 1 People's Hospital, Hubei University of Medicine.

Plasmid and primer. GV316-STAT3 plasmid, STAT3-specific siRNA and its control gene segment were all purchased from New Cycle Biotech Co., Ltd. (Wuhan, China).

Cell culture and transfection. SiHa cells were cultured at $37^{\circ} \mathrm{C}$ in a thermostat incubator containing $5 \% \mathrm{CO}_{2}$. Then, SiHa cells were inoculated on the 6-well plate, respectively, and when the cell adhesion degree reached $>90 \%$, the liposome transfection technique was performed according to the defined instructions of Lipofectamine 2000. At $6 \mathrm{~h}$ of transfection, the medium was replaced. After 24 h, G418 was added onto the medium and the culture was continued. Following the cell proliferation, we extracted the total protein and RNA to assay the expression of STAT3 using western blotting and RT-qPCR.

The mRNA expression of OCT4, SOX2 and NANOG using $q R T-P C R$. Cells in each group were collected after $48 \mathrm{~h}$ of transfection followed by extraction of total RNA of cells in each group using TRIzol reagent. Reverse transcription and gel electrophoresis was performed to obtain the cDNA according to the kit instructions. The mRNA expression of OCT4, SOX2 and $N A N O G$ were assayed using RT-qPCR after transfection. The primer sequences are shown in Table I.

Detection of the protein expression of OCT4, SOX2 and NANOG using western blotting. After the collection of transfected cells, the cells were treated using $100 \mu \mathrm{l}$ lysis buffer on ice for $30 \mathrm{~min}$, followed by centrifugation at $4^{\circ} \mathrm{C}$ at $11,500 \mathrm{x} g$ for $30 \mathrm{~min}$ to obtain the supernatant of the lysate. Then the supernatant served for detecting the protein concentration using BCA method. After the 10\% SDS-PAGE, the samples were transferred to PVDF membrane and blocked using 5\% skimmed milk powder prepared by TBST at room temperature for $1 \mathrm{~h}$. The membrane was incubated at $4^{\circ} \mathrm{C}$ overnight using rabbit monoclonal OCT4 antibody (dilution, 1:1,000; cat. no. ab18976); rabbit monoclonal SOX2 antibody (dilution, 1:1,000; cat. no. ab97959) and rabbit monoclonal NANOG antibody (dilution, 1:1,000; cat. no. ab80892) (all from Abcam, Cambridge, MA, USA). Then the membrane was washed three times using TBST, 5 min each time. The secondary goat anti-rabbit (HRP) IgG antibody (dilution, 1:2,000; cat. no. ab6721; Abcam, Cambridge, MA, USA) was added for $1 \mathrm{~h}$ of incubation followed by rinsing the membrane using TBST 3 times. Finally, color development was carried out for the membrane.

Culture of tumor sphere. Cells in the logarithm phase were digested and rinsed with PBS twice, followed by suspending the culture on the serum-free medium (DMEM:F12 = 1:1), in which $0.25 \mathrm{ml}$ serum-free fresh medium was added on a daily basis. The formation of tumor sphere was observed, photographed and recorded. After 10 days, the tumor sphere was collected and mechanically isolated for passaging (1:2). Fresh medium $(0.25 \mathrm{ml})$ was added into the medium each day. After 7 days, sub-culturing was performed to determine cell proliferation.

Immunohistochemistry. The pathological section of cervix was stained using SP, an immunohistochemical staining method was carried out as described below. A total of $30 \mathrm{ml} / 1$ $\mathrm{H}_{2} \mathrm{O}_{2}$ was used to deactivate the endogenous peroxidase and antigen retrieval was carried out in the boiled citrate acid buffer ( $\mathrm{pH}$ 6.0) in an autoclave. The section was blocked using regular goat serum for $30 \mathrm{~min}$ followed by incubation at $4^{\circ} \mathrm{C}$ overnight using polyclonal goat anti-human STAT3, OCT4, SOX2 and NANOG antibody. The section was incubated using biotin-labelled secondary antibody for $30 \mathrm{~min}$ and HRP-labelled streptavidin for another $30 \mathrm{~min}$ (Bioss Inc., Woburn, MA, USA). Then the section was treated with DAB for color development and hematoxylin for re-dyeing, followed by regular dehydration, clearing, drying and sealing. Then, the results were observed and analyzed under a light microscope (BX-42; Olympus, Tokyo, Japan). For the negative control group, the primary antibody was replaced by regular goat serum and the rest of the procedures were the same as above.

Statistical analysis. SPSS 19.0 (Chicago, IL, USA) was used for data analysis, and each experiment was repeated thrice. All experimental data are presented as mean \pm standard deviation. $\mathrm{t}$-test was performed for the comparison between the means of two samples. Statistical significance was set at $\mathrm{p}<0.05$.

\section{Results}

Construction and identification of STAT3 plasmid vector. Following gel electrophoresis, STAT3 obtained from 
Table I. Primers for RT-PCR and RT-qPCR.

\begin{tabular}{llc}
\hline Genes & \multicolumn{1}{c}{ Sequence (5'-3') } & Product size (bp) \\
\hline OCT4 & CGTGAAGCTGGAGAAGGAGAAGCTG & 247 \\
& CAAGGGCCGCAGCTTACACATGTTC & 170 \\
NANOG & CGCCCCCAGCAGACTTCACA & 164 \\
& CTCCTCTTTTGCACCCCTCCCATTT & 226 \\
GAPDH & AGTCCCAAAGGCAAACAACCCACTTC & \\
& ATCTGCTGGAGGCTGAGGTATTTCTGTCTC &
\end{tabular}

A

B

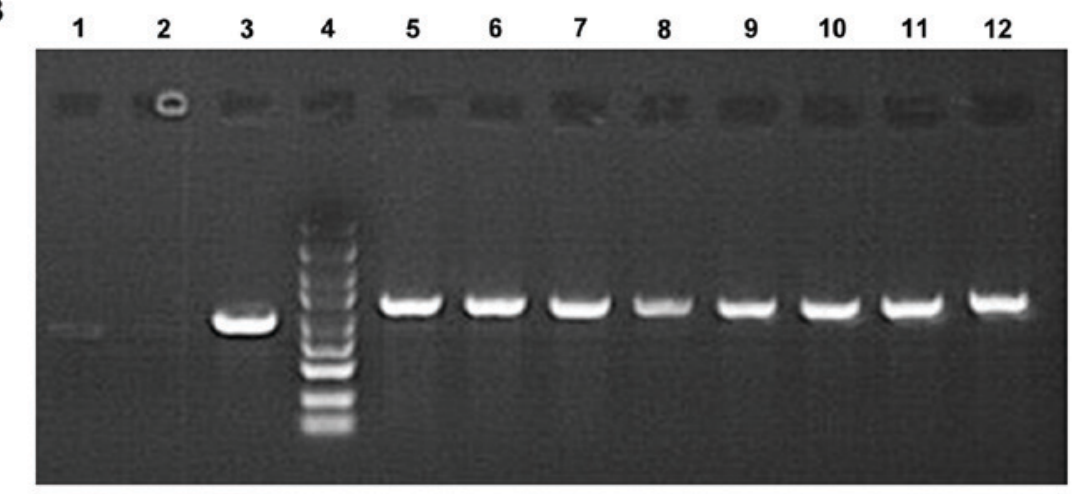

C
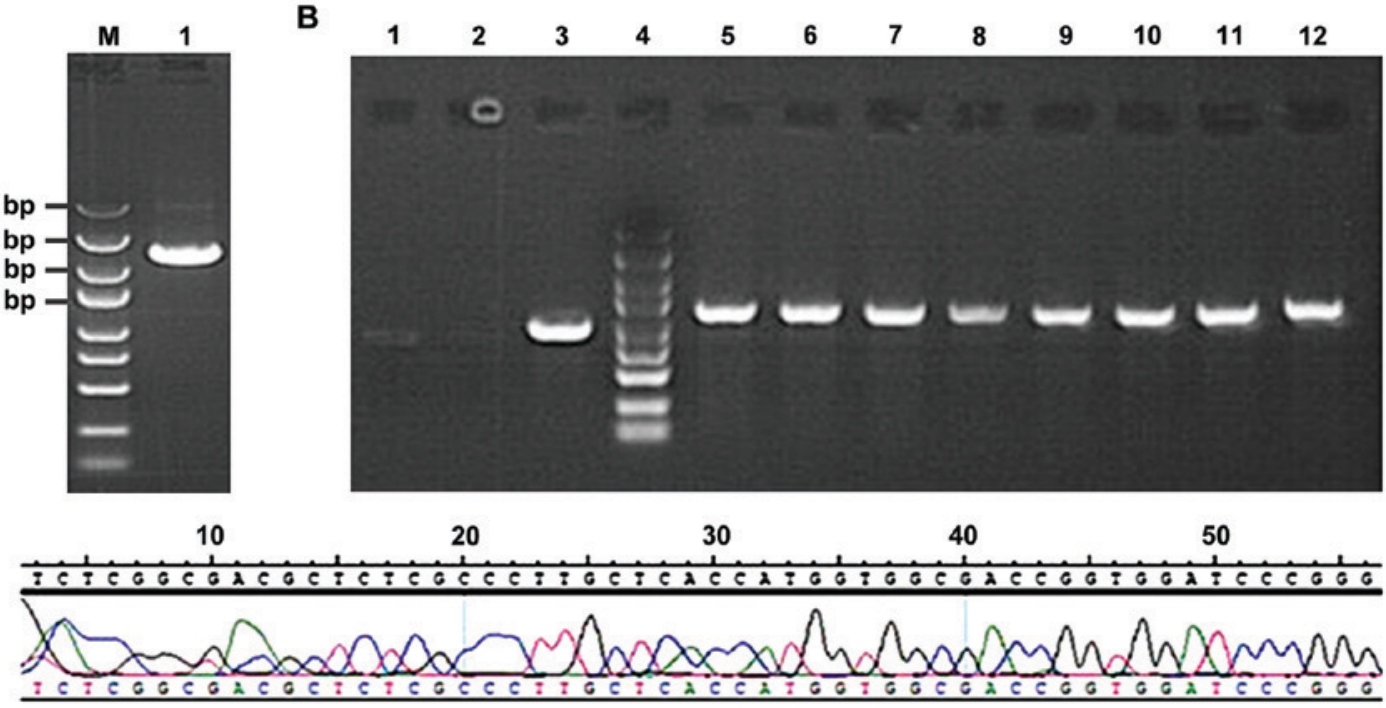

Figure 1. Agarose gel electrophoresis for PCR product of STAT3. (A) Lane 1, STAT3 PCR product; M for marker, DS5000; (B) lanes 1 and 2, negative control; lane 3, positive control; lane 4 Marker, DS5000; lanes 5-12, PCR products. (C) Positive clonal sequence of recombinant plasmid GV316-STAT3. STAT3, signal transducer and activator of transcription 3.

amplification using PCR (Fig. 1A) was bound with GV316 vector using T4 DNA ligase. After the transformation and LA plate-spread procedures, we selected the monoclonal genes for PCR and found the results were coincident with the anticipated $1265 \mathrm{bp}$, indicating that the binding was successfully completed (Fig. 1B). The sequencing results suggested that the clonal plasmid was inserted correctly and the quality was in accordance with the standard design (Fig. 1C).

Verification of STAT3 overexpression plasmid and siRNASTAT3 efficiency. The results of RT-qPCR and western blot analysis (Fig. 2) revealed that after the successful transfection of GV316-STAT3 plasmid, the mRNA and protein expression of STAT3 was significantly increased compared to that in the control group and empty plasmid group, indicating that STAT3 was successfully overexpressed.

Detection of the effect of STAT3 on the mRNA and protein expression of stem cell markers NANOG, OCT4 and SOX2, using western blot analysis and RT-qPCR. We employed the western blot analysis and RT-qPCR to assay the expression of NANOG, OCT4 and SOX2 (Figs. 3 and 4). Compared with those in the empty plasmid group and STAT3 overexpression group, the mRNA and protein expression of stem cell markers (NANOG, OCT4 and SOX2) in the siRNA-STAT3 group was significantly decreased with statistically significant difference $(\mathrm{P}<0.05)$. By contrast, the expression of NANOG, OCT4 and SOX2 in the STAT3 overexpression group was significantly increased compared to those in the empty plasmid group and siRNA-STAT3 group with statistically significant differences $(\mathrm{P}<0.05)$. The results showed that the expression of stem cell markers is concurrently accompanied with the overexpression of STAT3, indicating that there is a correlation between them, and STAT3 may affect the expression of stem cell markers (NANOG, OCT4 and SOX2). Thus, STAT3 can affect the biological characteristics of stem cells in cervical carcinoma through the stem cell markers (NANOG, OCT4 and SOX2).

Comparison of the formation rate of tumor spheres through the culture of tumor cells in serum-free medium. The formation rates of first generation tumor spheres in empty plasmid group, STAT3 overexpression group and siRNA-STAT3 group were $5.2 \pm 0.38,12.3 \pm 0.72$ and $2.3 \pm 0.012 \%$, respectively. After 

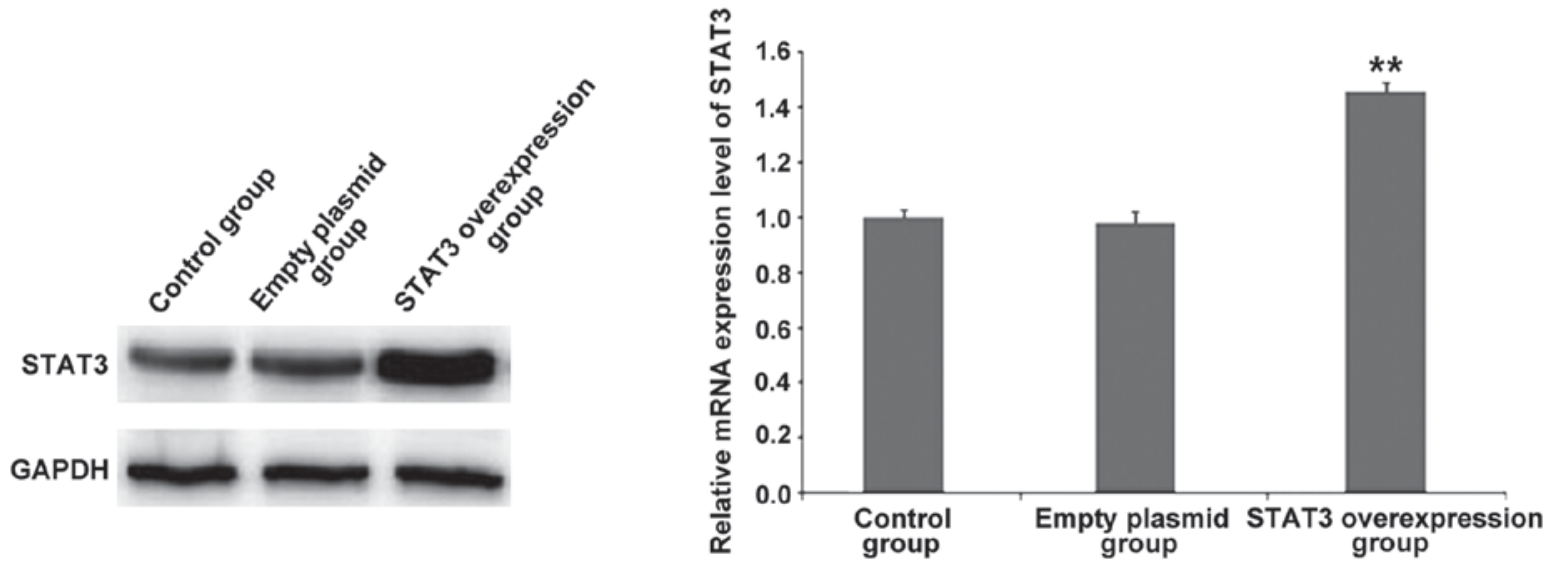

Figure 2. Detection of the mRNA and protein expression of STAT3 using RT-qPCR and western blotting in different groups. STAT3, signal transducer and activator of transcription $3 .{ }^{* *} \mathrm{P}<0.01$.

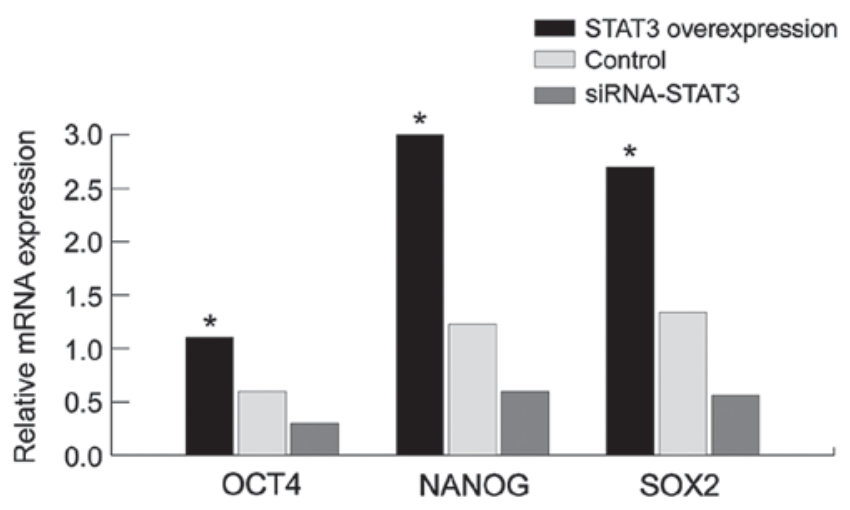

Figure 3. Detection of the mRNA expression of NANOG, OCT4 and SOX2 using RT-qPCR in STAT3 overexpression group and siRNA-STAT3 group. STAT3, signal transducer and activator of transcription $3 .{ }^{*} \mathrm{P}<0.05$.

3 weeks, the formation rate of tumor spheres in the STAT3 overexpression group was continuously increased, and relatively loose tumor spheres were found to be formed in the siRNA-STAT3 group ( $\mathrm{P}<0.05$; Fig. 5). In addition, the results of this study suggested that STAT3 overexpression can activate the tumor cells to form spheres, which, signifies at cellular level that STAT3 can affect the biological characteristics of stem cells in cervical carcinoma.

Expression of STAT3, NANOG, OCT4 and SOX2 in cervical carcinoma tissues and chronic cervicitis. STAT3, NANOG, OCT4 and SOX2 are mainly expressed in the nucleus of cervical carcinoma cells, and scarcely in the cytoplasm. The nuclei that are presented as tan or sepia-colored granules represent the positive staining which, however, is hardly to be found in the regular cervical squamous epithelial tissues, or only mildly stained area is evident (Fig. 6). The positive expression rates of STAT3, NANOG, OCT4 and SOX2 in the cervical carcinoma tissues and chronic cervicitis were respectively $6.45 \%(2 / 31)$ and $45.7 \%$ (16/35), $12.9 \%(4 / 31)$ and $57.1 \%(20 / 35), 9.67 \%(3 / 31)$ and $62.9 \%(22 / 35), 9.67 \%(3 / 31)$ and $54.3 \%(19 / 35), 9.67 \%(3 / 31)$ and $62.9 \%(22 / 35), 9.67 \%(3 / 31)$ and $54.3 \%$ (19/35). We found that the differences of the positive expression rate of STAT3, NANOG, OCT4 and SOX2 in cervical carcinoma and chronic cervicitis were statistically significant $(\mathrm{P}<0.05)$.

\section{Discussion}

In this study, to investigate the mutually dependent correlations among the proteins STAT3, NANOG, OCT4 and SOX2 and to confirm whether STAT3 was capable of regulating the biological characteristics of stem cells in cervical carcinoma by affecting the proteins NANOG, OCT4 and SOX2, we constructed the STAT3 overexpression plasmid (Figs. 1 and 2), which served as the basis for research into the regulatory effect of STAT3 on the biological characteristics of stem cells in cervical carcinoma. The results of western blotting and RT-qPCR revealed that the mRNA and protein expression of NANOG, OCT4 and SOX2 in the STAT3 overexpression group was significantly increased compared to those in the empty plasmid group and STAT3 low-expression group. However, when the STAT3 expression was inhibited using siRNA, the expression of NANOG, OCT4, and SOX2 was decreased with statistically significant differences $(\mathrm{P}<0.05)$. The result indicates that STAT3 can affect the mRNA and protein expressions of NANOG, OCT4 and SOX2, and that NANOG, OCT4 and SOX2 may be the key downstream proteins of STAT3.

To further verify whether STAT3 in the cell strain of cervical carcinoma regulated the biological characteristics of stem cells by affecting the proteins of NANOG, OCT4 and SOX2, we employed the tumor sphere suspension culture in serum-free medium and the results revealed that the highest formation rate of tumor sphere was found in the cervical carcinoma cells in the STAT3 overexpression group $(\mathrm{P}<0.05)$, and only loose tumor spheres or no formation of tumor spheres were found in the siRNA-STAT3 group, signifying that STAT3 overexpression can activate the formation of tumor spheres, and suggesting at a cellular level that STAT3 can affect the biological characteristics of stem cells in cervical carcinoma.

Furthermore, we detected the expression of STAT3, NANOG, OCT4 and SOX2 in clinical samples using immunohistochemistry. The results showed that the positive expression rates of STAT3, NANOG, OCT4 and SOX2 in the cervical carcinoma tissues and chronic cervicitis were respectively $6.45 \%(2 / 31)$ and $45.7 \%(16 / 35), 12.9 \%(4 / 31)$ and $57.1 \%$ $(20 / 35), 9.67 \%(3 / 31)$ and $62.9 \%(22 / 35), 9.67 \%(3 / 31)$ and $54.3 \%$ (19/35), 9.67\% (3/31) and 62.9\% (22/35), 9.67\% (3/31) 

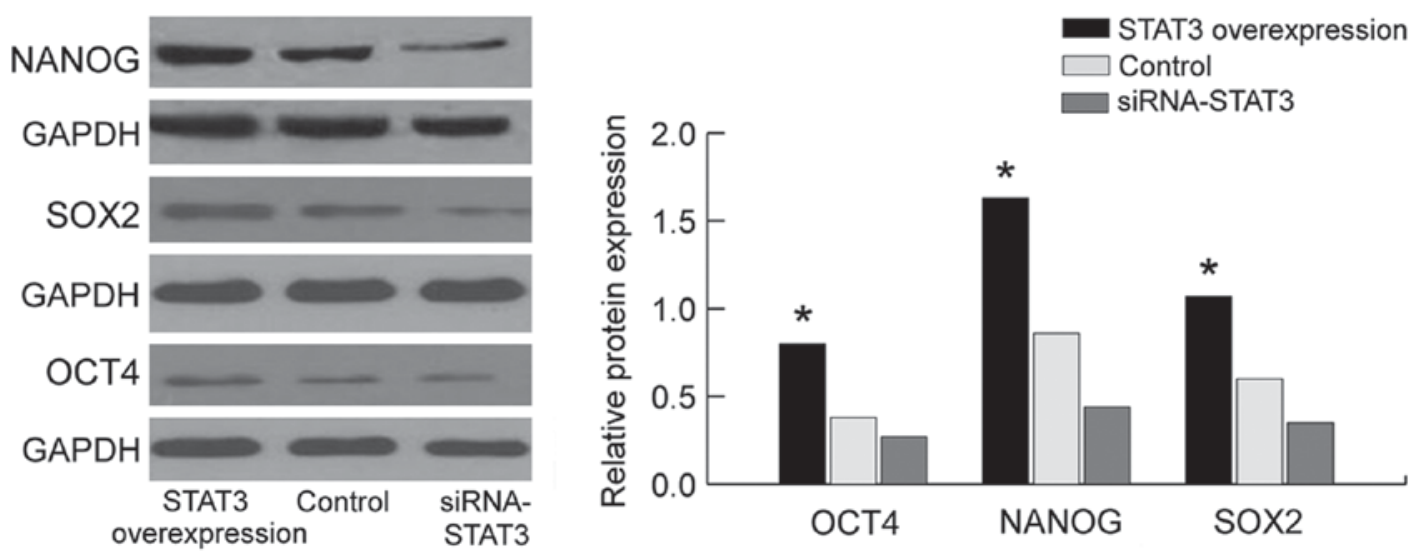

Figure 4. Protein expression of NANOG, OCT4 and SOX2 using western blotting in STAT3 overexpression group and siRNA-STAT3 group. STAT3, signal transducer and activator of transcription $3 .{ }^{*} \mathrm{P}<0.05$.
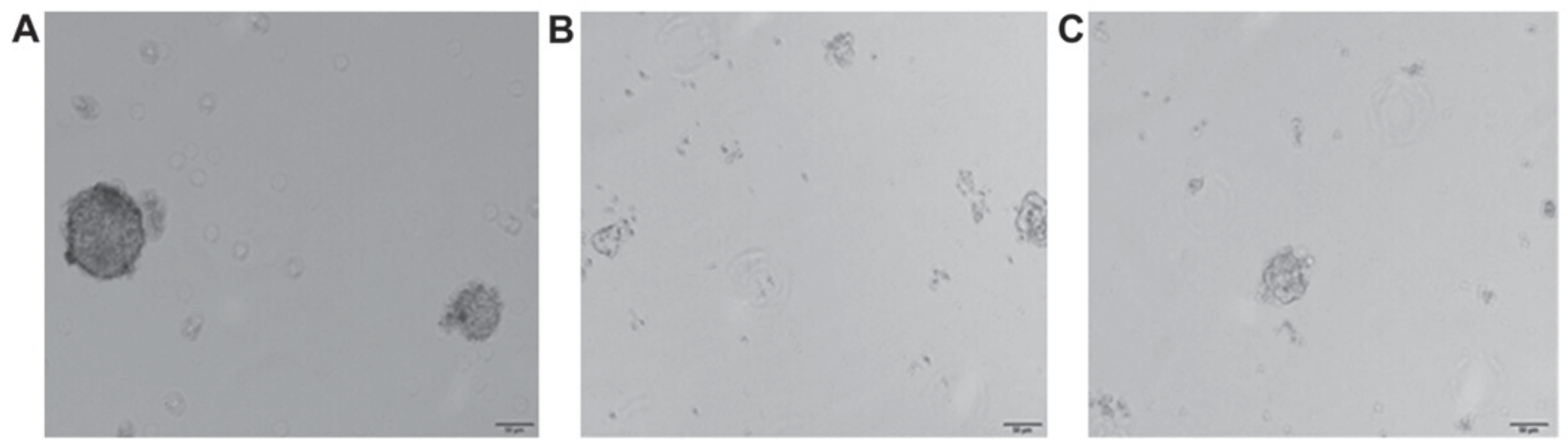

Figure 5. Influence of STAT3 on the formation of tumor spheres in cell strains of cervical carcinoma. (A) Empty plasmid group, (B) STAT3 overexpression group, and (C) siRNA-STAT3 group. STAT3, signal transducer and activator of transcription 3.
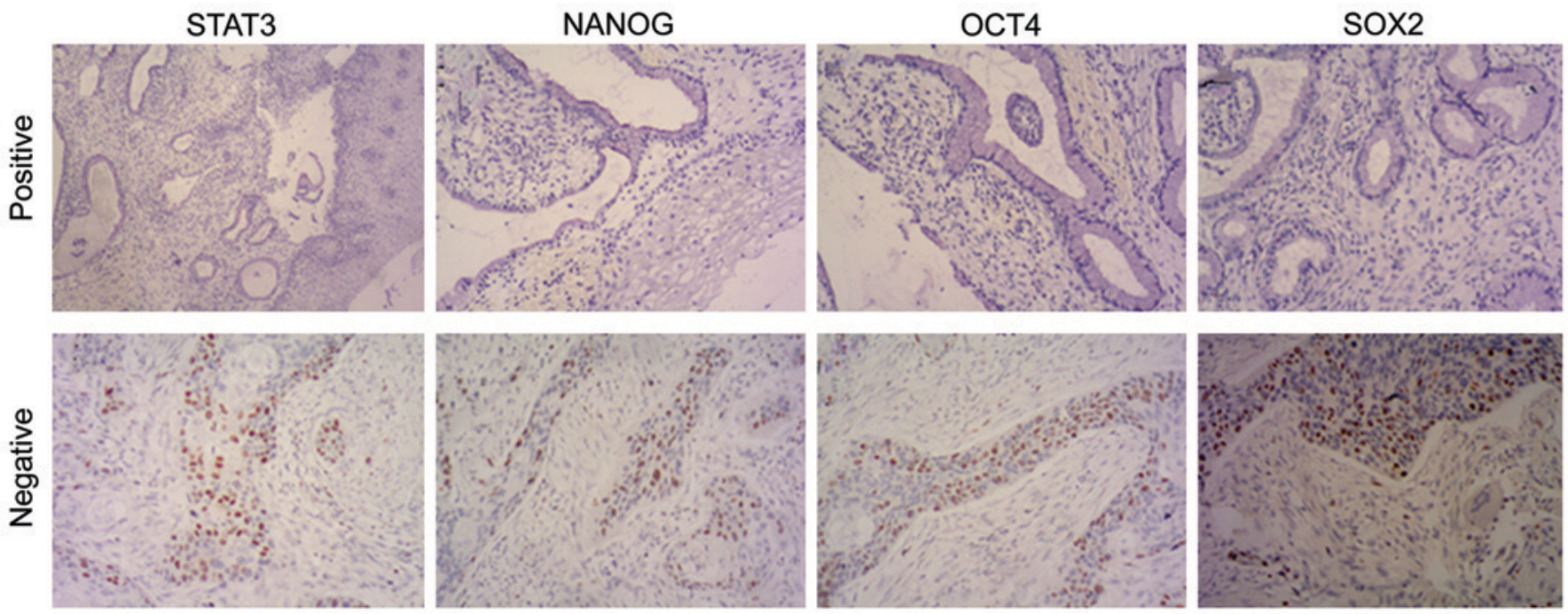

Figure 6. Expression of STAT3, NANOG, OCT4 and SOX2 in cervical carcinoma tissues (positive) and in chronic cervicitis tissues (negative). STAT3, signal transducer and activator of transcription 3.

and $54.3 \%$ (19/35). Thus, STAT3, NANOG, OCT4 and SOX2, mostly expressed in chronic cervicitis tissues, are presented as negative, while some expression of NANOG, OCT4 and SOX2 in all the levels of cervical carcinoma tissues is positive, which is coincident with the feature that tumor stem cells are partially expressed in the tumor tissues. Thus, the results of the clinical experiments in this study conform to those of the molecular biology experiments. 
Based on these cellular and molecular experiments, we subsequently investigated and analyzed the roles of the STAT3/NANOG pathway on the xenograft models of cervical carcinoma.

\section{References}

1. Feng D, Peng C, Li C, Zhou Y, Li M, Ling B, Wei H and Tian Z: Identification and characterization of cancer stem-like cells from primary carcinoma of the cervix uteri. Oncol Rep 22: 1129-1134, 2009.

2. Bortolomai I, Canevari S, Facetti I, De Cecco L, Castellano G, Zacchetti A, Alison MR and Miotti S: Tumor initiating cells: development and critical characterization of a model derived from the A431 carcinoma cell line forming spheres in suspension. Cell Cycle 9: 1194-1206, 2010.

3. Takebe N, Harris PJ, Warren RQ and Ivy SP: Targeting cancer stem cells by inhibiting Wnt, Notch, and Hedgehog pathways. Nat Rev Clin Oncol 8: 97-106, 2011.

4. Song L, Turkson J, Karras JG, Jove R and Haura EB: Activation of Stat 3 by receptor tyrosine kinases and cytokines regulates survival in human non-small cell carcinoma cells. Oncogene 22 4150-4165, 2003.

5. Zhang P, Li H, Yang B, Yang F, Zhang LL, Kong QY, Chen XY, Wu ML and Liu J: Biological significance and therapeutic implication of resveratrol-inhibited Wnt, Notch and STAT3 signaling in cervical cancer cells. Genes Cancer 5: 154-164, 2014.

6. Kruczyk M, Przanowski P, Dabrowski M, Swiatek-Machado K, Mieczkowski J, Wallerman O, Ronowicz A, Piotrowski A, Wadelius C, Kaminska B and Komorowski J: Integration of genome-wide of Stat 3 binding and epigenetic modification mapping with transcriptome reveals novel Stat3 target genes in glioma cells. Biochim Biophys Acta 1839: 1341-1350, 2014.

7. Yao J, Qian CJ, Ye B,Zhao ZQ, Wei J,Liang Y and Zhang X: Signal transducer and activator of transcription 3 signaling upregulates fascin via nuclear factor- $\kappa \mathrm{B}$ in gastric cancer: Implications in cell invasion and migration. Oncol Lett 7: 902-908, 2014.

8. Bao W, Wang HH, Tian FJ, He XY, Qiu MT, Wang JY, Zhang HJ, Wang LH and Wan XP: A TrkB-STAT3-miR-204-5p regulatory circuitry controls proliferation and invasion of endometrial carcinoma cells. Mol Cancer 12: 155, 2013.

9. Cheng GZ, Zhang WZ, Sun M, Wang Q, Coppola D, Mansour M, Xu LM, Costanzo C, Cheng JQ and Wang LH: Twist is transcriptionally induced by activation of STAT3 and mediates STAT3 oncogenic function. J Biol Chem 283: 14665-14673, 2008.
10. Qin A, Yu Q, Gao Y, Tan J, Huang H, Qiao Z and Qian W: Inhibition of STAT3/cyclinD1 pathway promotes chemotherapeutic sensitivity of colorectal caner. Biochem Biophys Res Commun 457: 681-687, 2015.

11. Takemoto S, Ushijima K, Kawano K, Yamaguchi T, Terada A, Fujiyoshi N, Nishio S, Tsuda N, Ijichi M, Kakuma T, et al: Expression of activated signal transducer and activator of transcription-3 predicts poor prognosis in cervical squamous-cell carcinoma. Br J Cancer 101: 967-972, 2009.

12. Devarajan E and Huang S: STAT3 as a central regulator of tumor metastases. Curr Mol Med 9: 626-633, 2009.

13. Cao C, Zhao G, Yu W, Xie X, Wang W, Yang R, Lv X and Liu D: Activation of STAT3 stimulates AHSP expression in K562 cells. Sci China Life Sci 57: 488-494, 2014.

14. Chambers I, Colby D, Robertson M, Nichols J, Lee S, Tweedie S and Smith A: Functional expression cloning of Nanog, a pluripotency sustaining factor in embryonic stem cells. Cell 113: 643-655, 2003.

15. Ye F, Zhou C, Cheng Q, Shen J and Chen H: Stem-cell-abundant proteins Nanog, Nucleostemin and Musashil are highly expressed in malignant cervical epithelial cells. BMC Cancer 8: 108, 2008.

16. Tsai LL, Yu CC, Chang YC, Yu CH and Chou MY: Markedly increased Oct 4 and Nanog expression correlates with cisplatin resistance in oral squamous cell carcinoma. J Oral Pathol Med 40: 621-628, 2011.

17. Wang Z, Oron E, Nelson B, Razis S and Ivanova N: Distinct lineage specification roles for NANOG, OCT4, and SOX2 in human embryonic stem cells. Cell Stem Cell 10: 440-454, 2012.

18. Chiou SH, Yu CC, Huang CY, Lin SC, Liu CJ, Tsai TH, Chou SH, Chien CS, Ku HH and Lo JF: Positive correlations of Oct-4 and Nanog in oral cancer stem-like cells and high-grade oral squamous cell carcinoma. Clin Cancer Res 14: 4085-4095, 2008.

19. Liu XF, Yang WT, Xu R, Liu JT and Zheng PS: Cervical cancer cells with positive Sox 2 expression exhibit the properties of cancer stem cells. PLoS One 28: 870-892, 2014.

20. Li SW, Wu XL, Dong CL, Xie XY, Wu JF and Zhang X: The differential expression of OCT4 isoforms in cervical carcinoma. PLoS One 10: $\mathrm{e} 0118033,2015$.

21. Bourguignon LY, Peyrollier K, Xia W and Gilad E: Hyaluronan-CD44 interaction activates stem cell marker Nanog, Stat-3-mediated MDR1 gene expression, and ankyrin-regulated multidrug efflux in breast and ovarian tumor cells. J Biol Chem 283: 17635-17651, 2008.

22. Do DV, Ueda J, Messerschmidt DM, Lorthongpanich C, Zhou Y, Feng B, Guo G, Lin PJ, Hossain MZ, Zhang W, et al: A genetic and developmental pathway from STAT3 to the OCT4-NANOG circuit is essential for maintenance of ICM lineages in vivo. Genes Dev 27: 1378-1390, 2013. 